Chapter 14

\title{
Optimal Vibrotactile Stimulation Activates the Parasympathetic Nervous System
}

\author{
Nelcy Hisao Hiraba, Motoharu Inoue, Takako Sato, \\ Satoshi Nishimura, Masaru Yamaoka, \\ Takaya Shimano, Ryuichi Sampei, Katuko Ebihara, \\ Hisako Ishii and Koichiro Ueda
}

Additional information is available at the end of the chapter

http://dx.doi.org/10.5772/51156

\section{Introduction}

We currently live in an environment that has become more and more stressful. Escaping from the stress in society through various activities (e.g., acupuncture, massage, listening to classic music or natural sounds, etc.) is important for our mental health. We previously reported that optimal facial vibrotactile stimulation (i.e., $89 \mathrm{~Hz}$ frequency and $1.9 \mu \mathrm{m}$ amplitude [89 Hz-S]) might activate the parasympathetic nervous system (Hiraba et al. 2008, 2011). Specifically, we showed that $89 \mathrm{~Hz}-\mathrm{S}$ stimulation of the face led to increased salivation and a feeling of mental well-being through parasympathetic activity based on functional near-infrared spectroscopy (fNIRS) oxyhaemoglobin (oxyHb) activity. Namely, brain blood flow (BBF) oxyHb in the frontal cortex was near zero (Hiraba et al. 2011). We investigated adaptation to the continuous use of vibrotactile stimuli for 4 or 5 days in the same subjects to determine whether this resulted in decreased salivation (Despopoulos and Silbernagel, 2003; Principles of Neural Science, 2000a). Then, we compared resting and stimulated salivation and investigated the most effective frequency for increasing salivary secretion. Increased salivation in normal subjects was defined as a difference between resting and stimulated salivation (Hiraba et al. 2011).

Furthermore, to study the mechanism of increased salivation evoked by vibrotactile stimulation, we recorded changes in heartbeat frequency and pupillary reflex during stimulation. We reported that pulse frequency changes during vibrotactile stimulation. A decrease in pulse frequency and a contraction in pupil diameter suggest parasympathetic activity (Prin- 
ciples of Neural Science, 2000b). We believe these reflexes are coordinated by a highly interconnected set of structures in the brainstem and forebrain that form a central autonomic network (Principles of Neural Science, 2000b).

We found that vibrotactile stimulation increased salivation, as reported by Hiraba et al. (2008). Furthermore, Hiraba et al. (2011) reported that increased salivation due to facial vibrotactile stimulation might be due to parasympathetic stimulation based on frontal cortex BBF measurements. Particularly, vibrotactile stimulation at $89 \mathrm{~Hz}-\mathrm{S}$ using a single motor was most effective in increasing salivation without adaptation following continuous daily use. We know that autonomic activity changes heart rate and pupil diameter. Thus, we believe that heart rate and pupil diameter measurements during $89 \mathrm{~Hz}-\mathrm{S}$ stimulation represent the effects of the autonomic nervous system. In this study, we demonstrated that $89 \mathrm{~Hz}-\mathrm{S}$ stimulation led to mental stability due to parasympathetic activity.

\section{Material and Methods}

\subsection{Vibrotactile stimulation apparatus}

The vibrotactile stimulation apparatus consisted of an oscillating body and a control unit, as shown in Hiraba et al. (2008) and Yamaoka et al. (2007). The oscillating body was composed of the following two parts: (1) a headphone headset equipped with vibrators in the positions of the bilateral microphones and (2) a vibration electric motor (VEM; Rekishin Japan Co., LE12AOG) covered in silicon rubber (polyethyl methacrylate, dental mucosa protective material; Shyofu Co.) for conglobating the stimulation parts and preventing VEM warming due to long periods of vibration (Hiraba et al. 2008).

We examined the amount of salivation during vibrotactile stimulation on the bilateral masseter muscle belly (on the parotid glands) and on bilateral parts of the submandibular angle (on the submandibular glands). We determined the amount of salivation using a dental cotton roll ( $1 \mathrm{~cm}$ width, $3 \mathrm{~cm}$ length) positioned at the opening of the secretory ducts (i.e., the right and left parotid glands and right and left submandibular and sublingual glands) during vibrotactile stimulation of the bilateral parotid and submandibular glands. The weights of the wet cotton rolls after 3 min of use were compared with their dry weights measured previously (Hiraba et al. 2008).

\subsection{Stimulating salivation and frontal cortex $B B F$}

We determined that a 3-min salivation measurement with a 5-min recovery time was sufficient from a previous experiment (Hiraba et al. 2008, Hiraba et al. 2011). Furthermore, salivation is most effectively induced by vibrotactile parotid gland stimulation at $89 \mathrm{~Hz}-\mathrm{S}$, which was used in this experiment. We examined adaptation to vibrotactile stimulation by monitoring changes in salivation during 4 or 5 continuous days using the same time schedule (i.e., 89 $\mathrm{Hz}-\mathrm{S}$ ). Frontal cortex recordings were acquired using a fNIRS OEG16 instrument (Spectratech, Inc., Shelton, CT, USA) during vibrotactile stimulation. We conducted salivation tests 
with 19 normal subjects (six males, 13 females; average age: 22 years) and resting-stimulation examinations for adaptation with 26 normal subjects (11 males and 15 females; average age: 25 years). We also performed fNIRS in eight normal subjects (six males, two females; average age: 22 years) to examine the effects of resting state and classical music (Mozart, Eine kleine Nachtmusik). This experiment was performed between 3 and $5 \mathrm{pm}$ in a temperaturecontrolled, quiet room, as described in previous papers (Hiraba et al. 2008, 2011).

\subsection{HRV analysis during vibrotactile stimulation}

We recorded changes in power-spectral analysis of heart rates (HRV; Heart Rate Variability module, AD Instruments, Japan, under the following conditions: (1) resting state; (2) $89 \mathrm{~Hz}-\mathrm{S}$ stimulation on the face ( $89 \mathrm{~Hz}-\mathrm{S}$ face); (3) listening to Mozart (Mozart); (4) Mozart + $89 \mathrm{~Hz}-\mathrm{S}$ on the face; (5) $89 \mathrm{~Hz}-\mathrm{S}$ on the nape of the neck (89 Hz-S neck); and (6) listening to noise (Noise), as shown in Figure 2E. A power-spectral analysis of HRV module data was conducted using the period histogram analysis program based on distribution of the length of the RR interval for $3 \mathrm{~min}$, and typical values during various stimuli were analysed in terms of the highest value (i.e., peak value) during the recording period. For example, Figure 1A shows RR intervals (n1, n2, n3, n4 ms, and so on,) on the electrocardiogram (ECG) during vibrotactile stimulation. Figure 1B shows a peak value example (1000 ms) during vibrotactile stimulation. Heart rates during rest and during various stimuli were recorded for $3 \mathrm{~min}$, and then analyses of 3-min HRV data were performed off-line. When heart rates were compared among the rest and various stimulation conditions, we used the RR-interval peak value (i.e., $1000 \mathrm{~ms}$ in this example) obtained from the power-spectra analysis. We conducted these examinations with 16 normal subjects (11 males, five females; average age: 25 years). This experiment was performed at 3 and $5 \mathrm{pm}$ in a quiet, temperature-controlled room.

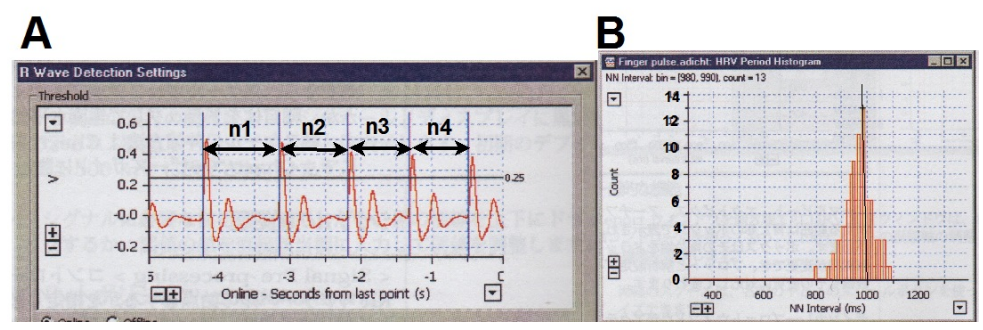

Figure 1. HRV module analysis. Method used to measure RR intervals ( $n 1, n 2, n 3$, n4, etc.) on ECG recordings (A) and frequency spectrum based on RR interval length over 3 min during $89 \mathrm{~Hz}-\mathrm{S}$ vibrotactile stimulation (B). Horizontal line indicates RR interval (ms), and vertical line indicates number. Note that the peak frequency spectrum was $1000 \mathrm{~ms}$ in this experiment.

\subsection{Pupillography during vibrotactile stimulation}

IRIS (Iriscorder, Hamamatsu Photonics Co., [Japan]) records transverse diameter and velocity reactions and can take a picture of the eyes by illuminating visible light (infrared radia- 
tion). The resulting image can record the condition of the iris and eyeball movement on the monitor. For example, when normal subjects are exposed to continuous light stimulation for $1 \mathrm{sec}$, we can obtain a pupillogram from the IRIS apparatus; constricted pupils indicate parasympathetic activity, and pupil dilation indicates sympathetic activity. Pupil diameter in normal subjects is generally $2-5 \mathrm{~mm}$, which changes under various adaptation conditions. We examined the transverse diameter and velocity of pupil constriction or dilatation after vibrotactile stimulation to explore changes in autonomic activity.
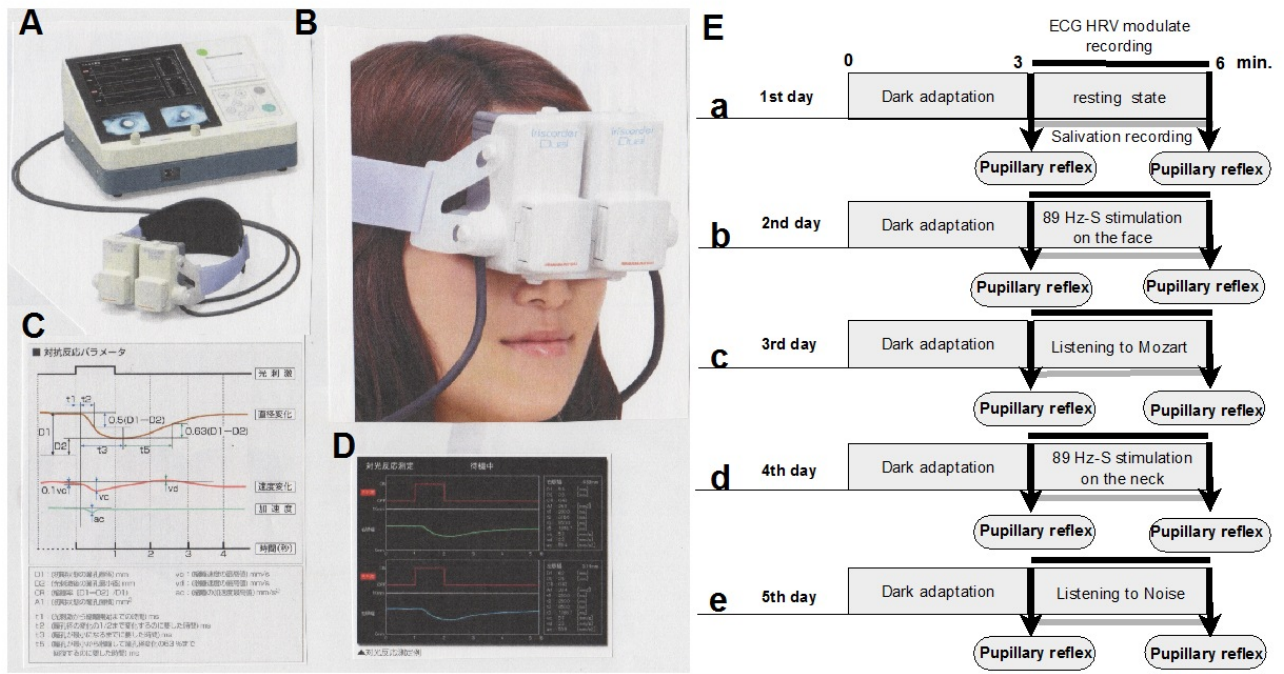

Figure 2. IRIS apparatus ( $A$ and $B$ ) and typical data acquired following light stimulation (C and D). IRIS records the bilateral pupillary reflex simultaneously. IRIS records the constricted and dilated pupil reflex for one second after light stimulation. Pupillary reflex parameters (e.g., most constricted pupil velocity) can be quantified. Experimental schedule (E).

Figure 2 shows the IRIS experimental apparatus used in Figures 2A and 2B. The pupillary light-reflex test was executed using an infrared pupillometer (Iriscorder C10641, Hamamatsu Photonics Co.), and pupil diameter in both the right and left eyes was measured after 3 min of rest or stimulation. Figure $2 \mathrm{E}$ shows the timeline of this experiment. Five particular experimental conditions were explored over 5 days; we recorded HRV modulation during the pupillary reflex adaptation test. However, only the right pupil was exposed to light stimulation, as shown in Figure 4. The pupillary test is non-invasive and enables real-time diagnosis. We examined the initial diameter (D1), minimum diameter (D2), constriction ratio (CR), time to total construction (T3), maximum velocity of constriction (VC), and maximum acceleration of constriction (AC) among the parameters obtained from the IRIS. Pupil diameter decreased with parasympathetic activity and increased with sympathetic activity. The IRIS records pupil parameters of the right and left eyes simultaneously. However, we adopted parameters from the right pupil because data from both sides were similar, as shown in Figure 4 . We conducted these examinations with eight normal subjects (six males, two 
females; average age: 25 years). This experiment was performed at 3 and $5 \mathrm{pm}$ in a quiet, temperature-controlled room. Furthermore, one parameter was explored each experimental day because we obtained information from adaptation to light stimulation, as shown by the experimental schedule in Figure 2E.

\section{Results}

\subsection{Vibrotactile stimulation of the parotid and submandibular glands}

We examined differences between vibrotactile stimulation of the parotid and submandibular glands. We found that the most effective frequency to induce salivation was $89 \mathrm{~Hz}-\mathrm{S}$ regardless of whether vibrotactile stimulation was delivered to the parotid or submandibular glands, as shown in previous paper (Hiraba et al. 2011).

Because patients with hyposalivation often have psychiatric disorders, we conducted an experiment to realistically approximate natural conditions. We examined whether effective salivation occurred continuously when vibrotactile stimulation was performed daily. Specifically, we used the $89 \mathrm{~Hz}$-S frequency with a single motor from the previous experiment. None of the glands (i.e., right and left parotid glands or right submandibular and sublingual glands) showed a reduced response. Regression curves for each gland showed non-adaptation to continuous stimulation; instead they showed parallel or increasing curves, indicating that continuous use of this apparatus should not be problematic, as shown in a previous paper (Hiraba et al. 2011).

\subsection{Relationship between stimulation and fNIRS activity}

The OEG16 spectroscope was used to record BBF haemoglobin concentration from areas in the frontal cortex using 16 channels. We determined the oxy $\mathrm{Hb}$ concentration schema evoked by $89 \mathrm{~Hz}$-S vibrotactile stimulation by analysing 16 channels. The results showed very weak oxyHb concentrations (i.e., near zero) during $89 \mathrm{~Hz}-\mathrm{S}$ vibrotactile stimulation. Changes in $\mathrm{oxyHb}$, deoxyhaemoglobin (deoxyHb), and total haemoglobin (totalHb) concentrations during salivation measurements at rest and for each vibrotactile stimulation frequency were measured. As shown in a previous paper (Hiraba et al. 2011), changes during the following six conditions were measured: (1) resting; (2) $89 \mathrm{~Hz}-\mathrm{S}$ vibrotactile stimulation; (3) $89 \mathrm{~Hz}-\mathrm{D}$ (89 $\mathrm{Hz}-\mathrm{D}, 89 \mathrm{~Hz}$ frequency with double motors, 3.5- $\mu$ m amplitude); (4) $114 \mathrm{~Hz}-\mathrm{S}$; (5) $114 \mathrm{~Hz}-\mathrm{D}$ (114 Hz-D, $114 \mathrm{~Hz}$ frequency with double motors, 3.5- $\mu \mathrm{m}$ amplitude); and (6) "A-" phonation. Each wave was recorded for $3 \mathrm{~min}$, and each 2-min vibrotactile stimulus is shown between the vertical lines (Fig. 6B and Fig. 4 in the previous paper, 2011). Although each wave measured during resting salivation, at $114 \mathrm{~Hz}-\mathrm{D}$, and during "A-" phonation showed increased activity, the $89 \mathrm{~Hz}-\mathrm{D}$ and $114 \mathrm{~Hz}-\mathrm{S}$ vibrotactile stimuli decreased activity. However, vibrotactile stimulation at $89 \mathrm{~Hz}-\mathrm{S}$ showed a value of almost zero. Particularly, when we focused on oxy $\mathrm{Hb}$ changes based on these results, increased oxyHb occurred during " $\mathrm{A}-$ " phonation, the resting condition, and at $114 \mathrm{~Hz}-\mathrm{D}$ vibrotactile stimulation, whereas a decrease in oxyHb was observed during vibrotactile stimulation at $114 \mathrm{~Hz}-\mathrm{S}$ and at $89 \mathrm{~Hz}-\mathrm{D}$. However, oxyHb con- 
centration during vibrotactile stimulation at $89 \mathrm{~Hz}-\mathrm{D}$ was almost zero, as were all other data $(\mathrm{oxyHb}$, deoxyHb, totalHb). From these results, we computed oxyHb integral rates over 2 min, as shown by the area between the longitudinal bars (Fig. 6B and Fig. 4 in the previous paper, 2011).

Furthermore, we examined integral rates while subjects listened to classical music for 2 min. We divided the subjects into two groups: (1) one group that disliked listening to classical music and (2) one group that enjoyed listening to classical music. Although the subjects who enjoyed the music did not show a larger spread of values, the former did. Specifically, vibrotactile stimulation at $89 \mathrm{~Hz}-\mathrm{S}$ led to a small, similar value spread. All integral rates during the vibrotactile stimulation at $89 \mathrm{~Hz}-\mathrm{S}$ and listening to classical music showed similar averages and standard deviations (SDs), as shown in Figure 6B.

\subsection{Total salivation during vibrotactile stimulation}

During facial $89 \mathrm{~Hz}-\mathrm{S}$ stimulation, values of total salivation and salivation in the parotid or submandibular and sublingual glands were examined in comparison with resting salivation values. Submandibular and sublingual gland total salivation valuesincreased; however, parotid gland salivation values were similar, as shown in Figure 3. Parotid gland salivation values were $0.15 \pm 0.12 \mathrm{ml}$ on both sides during rest and $0.14 \pm 0.12 \mathrm{ml}$ under the $89 \mathrm{~Hz}-\mathrm{S}$ stimulation condition. Salivation values of submandibular and sublingual glands on both sides was $0.79 \pm 0.44 \mathrm{ml}$ during rest and $1.00 \pm 0.58 \mathrm{ml}$ under the $89 \mathrm{~Hz}-\mathrm{S}$ condition $(p<0.01$; Wilcoxon singed-rank test, two-tailed). Total gland salivation values on both sides were $0.91 \pm 0.55 \mathrm{ml}$ during rest and $1.16 \pm 0.60 \mathrm{ml}$ under the $89 \mathrm{~Hz}-\mathrm{S}$ condition $(\mathrm{p}<0.01)$. Particularly, although parotid gland salivation was similar between the resting and $89 \mathrm{~Hz}-\mathrm{S}$ conditions, that in the submandibular and sublingual glands showed an absolute increase between the resting and $89 \mathrm{~Hz}-\mathrm{S}$ conditions, as shown in Figure 3.

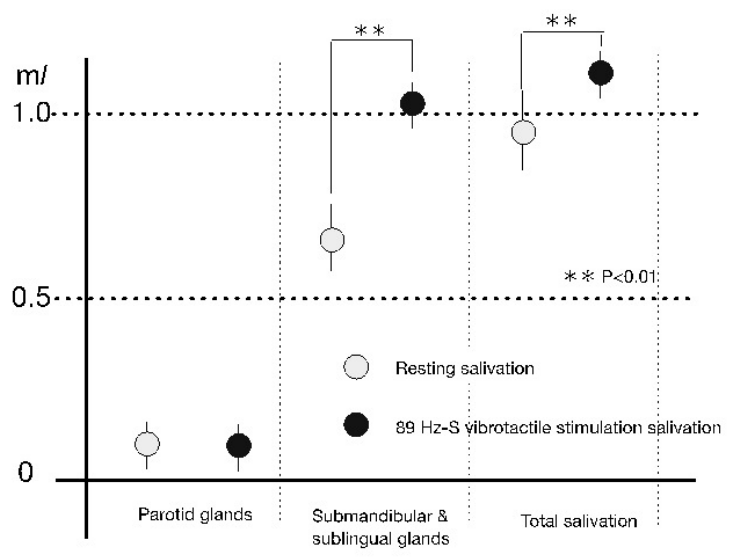

Figure 3. Salivation during the resting condition and $89 \mathrm{~Hz}-\mathrm{S}$ vibrotactile stimulation over 3 min. Wilcoxon signedrank test (two-tailed), $P<0.01$. 


\subsection{Pupillary reflex after vibrotactile stimulation}

Among the parameters obtained from the IRIS, we examined D1, D2, CR, T3, VC, and AC, as shown in Figures $4 \mathrm{~A}$ and $4 \mathrm{~B}$. The pupillary light reflex showed significantly decreased D1, D2, and T3 compared with the resting state. Furthermore, the pupillary light reflex showed increased AC, as shown in Figure 4B. Data from the right and left pupils were similar following light stimulation, as shown in Figure 4B. Thus, we employed data from the right pupillary reflex for data analysis.

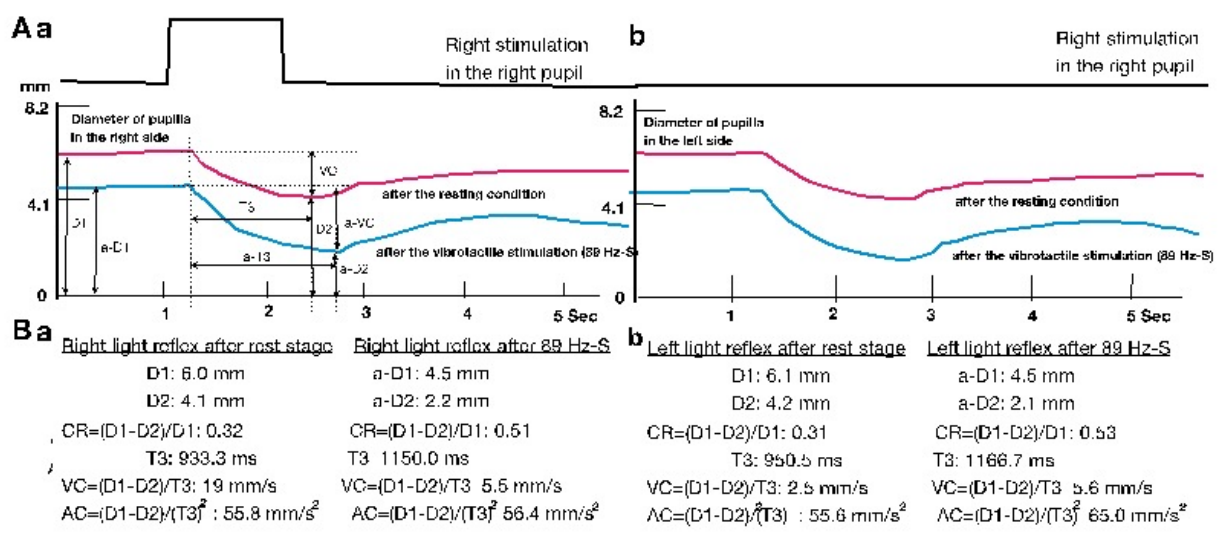

Figure 4. Typical example of data from the pupillary reflex. Right pupillary reflex data after light stimulation in the right pupil (A-a and B-a) and left pupillary reflex data after light stimulation in the left pupil (A-b and B-b).

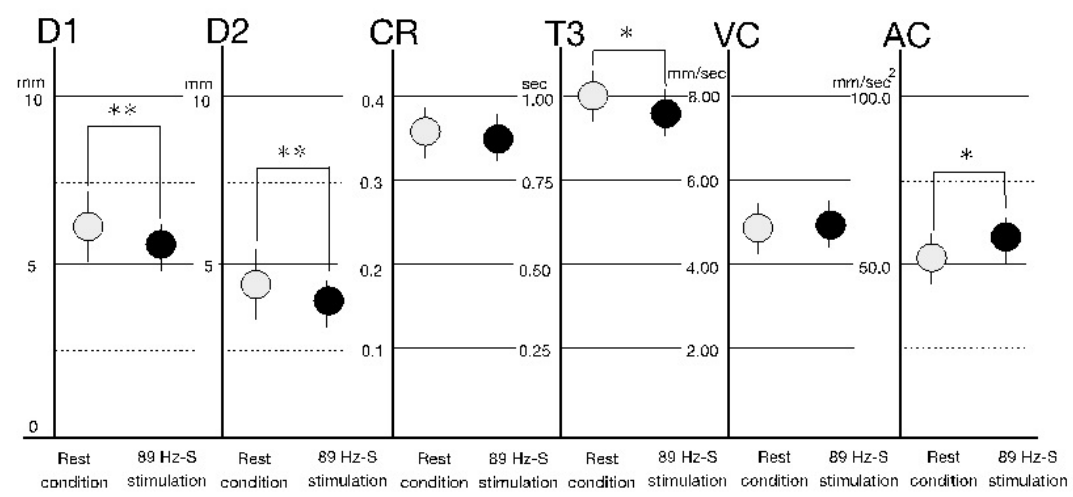

Figure 5. Effect of the pupillary reflex following right-side light stimulation between the resting condition and $89 \mathrm{~Hz}-\mathrm{S}$ vibrotactile stimulation. 
We analysed right pupillary reflex data from eight normal subjects, as shown in Figure 5. D1 was $6.15 \pm 0.64 \mathrm{~mm}$ under the resting condition and $5.20 \pm 1.12 \mathrm{~mm}$ under the $89 \mathrm{~Hz}-\mathrm{S}$ condition ( $p<0.01$ : Wilcoxon signed-rank test, two-tailed). D2 was $4.03 \pm 0.79 \mathrm{~mm}$ under the resting condition and $3.47 \pm 0.84 \mathrm{~mm}$ under the $89 \mathrm{~Hz}$-S condition $(p<0.01)$. CR was $0.37 \pm 0.10$ ms under rest and $0.35 \pm 0.09$ under the $89 \mathrm{~Hz}-\mathrm{S}$ condition. T3 was $1.089 \pm 0.094 \mathrm{~ms}$ under rest and $0.973 \pm 0.175 \mathrm{~ms}$ under the $89 \mathrm{~Hz}-\mathrm{S}$ condition $(p<0.05)$. VC was $4.90 \pm 0.95 \mathrm{~mm} / \mathrm{s}$ under rest and $5.03 \pm 0.89 \mathrm{~mm} / \mathrm{s}$ under the $89 \mathrm{~Hz}-\mathrm{S}$ condition. AC was $52.4 \pm 16.8 \mathrm{~mm} / \mathrm{s}^{2}$ under rest and $56.7 \pm 17.0 \mathrm{~mm} / \mathrm{s}^{2}$ under the $89 \mathrm{~Hz}-\mathrm{S}$ condition $(p<0.05)$. Of particular note, D1, D2, and T3 showed an absolute decrease between the resting state and $89 \mathrm{~Hz}-\mathrm{S}$ stimulation, and AC increased between the resting and $89 \mathrm{~Hz}-\mathrm{S}$ conditions. CR and VC did not change between the resting and $89 \mathrm{~Hz}-\mathrm{S}$ conditions, as shown in Figure 5.

\subsection{Analysis of HRV during vibrotactile stimulation}

We recorded typical heart rate changes and performed a power-spectral analysis (HRV module, AD Instruments, Japan) under the following six conditions: (1) resting state; (2) 89 Hz-S face; (3) listening to Mozart (Mozart); (4) Mozart + 89 Hz-S face; (5) 89 Hz-S neck; and (6) listening to noise (Noise), as shown in Figure 6. For example, Mozart $+89 \mathrm{~Hz}-\mathrm{S}$ face "pulse (+)" indicates that participants were listening to Mozart classical music while receiving $89 \mathrm{~Hz}$-S vibrotactile stimulation on the face. When comparing heart rates between the rest state and under various stimuli, we used the RR interval peak value from the powerspectral analysis (Fig. 6A and 6C). RR interval peak values (ms) from the power-spectral analysis were compared. The values were as follows: resting state, $757.5 \pm 57.0 \mathrm{~ms} ; 89 \mathrm{~Hz}$ S face, $905.1 \pm 189.5$ ms, Mozart, $771.7 \pm 86.7$ ms; Mozart + 89 Hz-S face, $875.3 \pm 188.3$ ms; $89 \mathrm{~Hz}-\mathrm{S}$ neck, $901.7 \pm 188.4 \mathrm{~ms}$; and Noise, $831.7 \pm 114.6 \mathrm{~ms}$ (Fig. 6C). Significant differences were observed between resting state and $89 \mathrm{~Hz}-\mathrm{S}$ face (paired $t$-test, $P<0.01$ ) and between resting state and Mozart $+89 \mathrm{~Hz}-\mathrm{S}$ face, $89 \mathrm{~Hz}$-S neck, and Noise (paired $t$-test, $P<$ 0.05; Fig. 6C).

The resting-state peak value had the lowest frequency. The Mozart-listening peak value was closest to the resting-state value, which might be because the majority of subjects disliked listening to classical music (three subjects favourite music was classical, and seven people reported classical music was not their favourite), as shown in Figure 6C. The $89 \mathrm{~Hz}-\mathrm{S}$ stimulation led to the highest heart-beat frequency in comparison with the resting condition, as shown in Figures 6A and 6C. However, $89 \mathrm{~Hz}-\mathrm{S}$ face stimulation was effective in many subjects, as $89 \mathrm{~Hz}-\mathrm{S}$ face had the smallest SD, as shown in Figure 6C. On the other hand, heart rates during the resting condition and while listening to noise were similar. We generated noise with fractioned foam polystyrene. Many subjects may have felt discomfort due to the noise; however, we believe that discomfort induced by this noise was unlikely. 


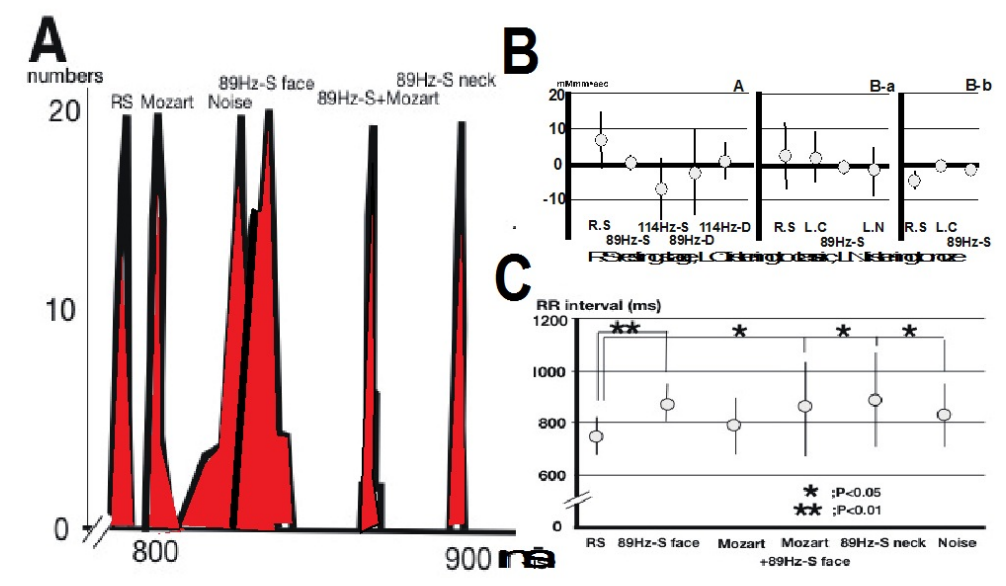

Figure 6. Changes in power spectrums (A) and HRV modulation (C) during various stimuli. B. fNIRS OxyHb concentration during various stimuli (this graph was described in a previous article, Hiraba et al. 2011). RS, 89 Hz-S face, Mozart, Mozart + $89 \mathrm{~Hz}-\mathrm{S}$ face, $89 \mathrm{~Hz}-\mathrm{S}$ neck, and Noise indicate $89 \mathrm{~Hz}-\mathrm{S}$ on the face, listening to Mozart, both listening to Mozart and $89 \mathrm{~Hz}-\mathrm{S}$ vibrotactile stimulation on the face, $89 \mathrm{~Hz}-\mathrm{S}$ vibrotactile stimulation on the nape of the neck, and listening to noise, respectively. There were significant differences between RS and $89 \mathrm{~Hz}$-S face (paired $t$-test, $P<0.01$ ), between RS and Mozart + $89 \mathrm{~Hz}-\mathrm{S}$ face, and between $89 \mathrm{~Hz}-\mathrm{S}$ neck and Noise (paired $t$-test, $P<0.05$ ).

\section{Discussion}

\subsection{Relaxation produced by $89 \mathrm{~Hz}-\mathrm{S}$ vibrotactile stimulation}

We reported that $89 \mathrm{~Hz}-\mathrm{S}$ vibrotactile stimulation evoked rest and increased salivation, as shown in previous papers (Hiraba et al. 2008, 2011). We further investigated increased salivation during $89 \mathrm{~Hz}$-S. We were the first to show that increased salivation during $89 \mathrm{~Hz}-\mathrm{S}$ stimulation was due to increased salivation from the submandibular and sublingual glands but not from the parotid glands, as shown in Figure 3. We knew that the amylase-rich parotid glands were principally responsible for the increased salivation. Salivation also occurs during mechanical stimulation during mastication when eating (Matuo, 2003). In addition to hunger- and mastication-induced salivation, salivation was also increased through $89 \mathrm{~Hz}-\mathrm{S}$ vibrotactile stimulation of the facial and intraoral structures. This increased salivation may be different from salivation produced by hunger, as increased salivation during $89 \mathrm{~Hz}-\mathrm{S}$ caused salivation in the submandibular and sublingual glands. In particular, increased salivation evoked by $89 \mathrm{~Hz}-\mathrm{S}$ vibrotactile stimulation may be due to somatosensory input from the facial skin and intraoral cavity. Vibrotactile stimulation at $89 \mathrm{~Hz}-\mathrm{S}$ may evoke a different perception from masticatory mechanical stimuli. 
The frontal cortex is associated with cognitive function, including memory, attention, abstract reasoning, and higher cognitive processes (Principles of Neural Science, 2000a). We recorded changes in frontal cortex BBF to examine typical changes in fNIRS parameters based on increased oxyHb and totalHb and decreased deoxyHb, as reported by Sakatani et al. (2006). The effect of $89 \mathrm{~Hz}-\mathrm{S}$ vibrotactile stimulation was almost zero for oxyHb, deoxyHb, and totalHb, as shown in a previous paper (Hiraba et al. 2011). The fNIRS activity focuses on excitatory behaviours that increase oxyHb. In animal experiments, changes in oxyHb and $\mathrm{BBF}$ are related, and fNIRS activity changes in oxyHb are used as a marker of neuronal activity (Hoshi et al. 2001). Thus, changes in oxyHb produced by $89 \mathrm{~Hz}-\mathrm{S}$ vibrotactile stimulation may indicate mental stability. This may be due to the trend in oxyHb concentration between the $89 \mathrm{~Hz}-\mathrm{S}$ vibrotactile stimulation in subjects who liked to listen to classical music (Fig. 6B). People relax when they listen to classical music, so we think that $89 \mathrm{~Hz}-\mathrm{S}$ vibrotactile stimulation elicits excitation of the parasympathetic system. In particular, although the $89 \mathrm{~Hz}-\mathrm{S}$ vibrotactile stimulation always led to parasympathetic excitation, listening to classical music caused different activity depending on music preference (Fig. 6B). Those subjects who enjoyed Mozart classical music found it relaxing, whereas those who disliked it perceived it as noise. However, $89 \mathrm{~Hz}-\mathrm{S}$ vibrotactile stimulation may lead to a balanced mental condition, regardless of preference. This phenomenon suggests that the effect caused by the $89 \mathrm{~Hz}-\mathrm{S}$ vibrotactile stimulation and the feeling experienced by those listening to Mozart who enjoyed it may be the same. Thus, we suggest that these feelings were produced by parasympathetic activity. We further investigated pupillary reflex and heart rate during 89 Hz-S stimulation.

\subsection{Parasympathetic effect produced by $89 \mathrm{~Hz}-\mathrm{S}$ vibrotactile stimulation}

Our heartbeat increases when we are frightened (Principles of Neural Science, 2000b). The parasympathetic nervous system is responsible for rest, digestion, basal heart rate maintenance, respiration, and metabolism under normal resting conditions (Principles of Neural Science, 2000b). We examined parasympathetic effects by observing changes in the amount of salivation, HRV modulation of heart rate, and pupillary reflex induced by light stimulation during various stimuli.

We verified increased salivation induced by $89 \mathrm{~Hz}-\mathrm{S}$ vibrotactile stimulation; the higher RR frequency was induced by the $89 \mathrm{~Hz}-\mathrm{S}$ neck stimulation (Fig. 6C), and the greatest pupil contraction following light stimulation was induced by $89 \mathrm{~Hz}-\mathrm{S}$ vibrotactile stimulation (Fig. 5). Furthermore, the $89 \mathrm{~Hz}-\mathrm{S}$ face stimulation increased salivation the most (Fig. 3). Specifically, increased salivation was due to saliva secretion by the submandibular and sublingual glands but not the parotid glands. This was likely not due to hunger because the amount of salivation in the parotid glands did not increase. From these results, as for $89 \mathrm{~Hz}-\mathrm{S}$ neck, the relaxation effect might be big, however the salivated promotion effect was very weak. Conversely, the difference between pupil diameter before (D1) and after (D2) light stimulation during the resting condition was great, but the difference between pupil diameter before (aD1) and after (a-D2) light stimulation during $89 \mathrm{~Hz}-\mathrm{S}$ face was small, as shown in Figure 4. Furthermore, as shown in Figure 5, the AC (acceleration) was significantly different between 
the resting and the $89 \mathrm{~Hz}-\mathrm{S}$ conditions $(P<0.05)$. These results suggested that the $89 \mathrm{~Hz}-\mathrm{S}$ vibrotactile stimulation may elicit parasympathetic activity and greater pupil acceleration. This is likely because parasympathetic activity was stimulated by the $89 \mathrm{~Hz}-\mathrm{S}$ face stimulation. Furthermore, vibrotactile stimulation at $89 \mathrm{~Hz}-\mathrm{S}$ also led to parasympathetic activity.

\subsection{Autonomic activity and anatomical projections in the central nervous system}

We examined parasympathetic activity in three organs. Autonomic function must ultimately be coordinated for adaptation to environmental changes. The autonomic nervous system is composed of visceral sensory and motor system; the visceral reflexes are controlled by various local circuits in the brainstem and spinal cord. These reflexes are regulated by networks of central autonomic control nuclei in the brainstem, hypothalamus, and forebrain and are not undervoluntary control, nor do they impingeon consciousness, with a few exceptions(Principles of Neural Science, 2000b). The pupillodilator muscle in the iris (pupil diameter), salivary glands, and heart rate are driven by sympathetic and parasympathetic nerves. Pupil diameter contracts, heart rate elongates, and salivation increases due to parasympathetic nerve activity. We also believe that changes in the frontal cortex BBF may represent autonomic activity. This coordination is carried out by a highly interconnected set of structures in the brainstem and forebrain that form a central autonomic network. The key component of this network is initiated by integrated information from the parabrachial nucleus of the solitary tract and trigeminal sensory complex in the brainstem. These nuclei receive inputs from somatosensory and visceral afferents of the trigeminal, facial, glossopharyngeal, and vagus nerves and then use the information to modulate autonomic function. The somatosensory and visceral sensory outputs from the trigeminal and solitary nuclei are relayed to the forebrain and amygdala by the parabrachial nucleus, which is important for behavioural responses to somatosensory, taste, and other visceral sensations (Principles of Neural Science, 2000b). Information arriving in the amygdala leads to sensations of pleasure and pain. In contrast, the parabrachial nucleus is a taste-sensation relay nucleus in rats (Scott and Small, 2009), and the rodent parabrachial nucleus sends integral limbic and reward system information (Yamamoto et al. 2009). Although their functions in humans are unknown, we think that these nuclei may play roles as relay nuclei of the autonomic system. On the other hand, we showed that a projection from the trigeminal sensory complex (e.g., the parabrachial nucleus) can also record the response to tactile stimuli from facial skin (Chiang et al. 1994). Furthermore, somatosensory information projects to the primary somatosensory cortex and is then relayed to the frontal cortex via the parietal association area (Handbook of Neuropsychology, 1994).

What does BBF activity in the frontal cortex indicate? We think that the information transmitted via the parabrachial nucleus dominates by way of the parietal association area. Thus, information in the frontal cortex is assumed to arrive via the parabrachial nucleus. The hypothalamus is the centre of the autonomic system. We perceive emotional experiences such as fear, pleasure, and contentment, and these perceptions reflect the interplay between higher brain centres and sub-cortical regions such as the hypothalamus and amygdala (Principles of Neural Science, 2000a). Patients in whom the prefrontal cortex or the cingulate gyrus has been removed are no longer bothered by pain but exhibit appropriate autonomic reactions; howev- 
er, the sensation is not perceived as a powerfully unpleasant experience (Principles of Neural Science, 2000a). Furthermore, the anatomical connections between the amygdala and the temporal (cingulate gyrus) and frontal (prefrontal) association cortices provide the means by which visceral and somatosensory sensations trigger a rich assortment of associations or the cognitive interpretation of emotional states (Principles of Neural Science, 2000b).

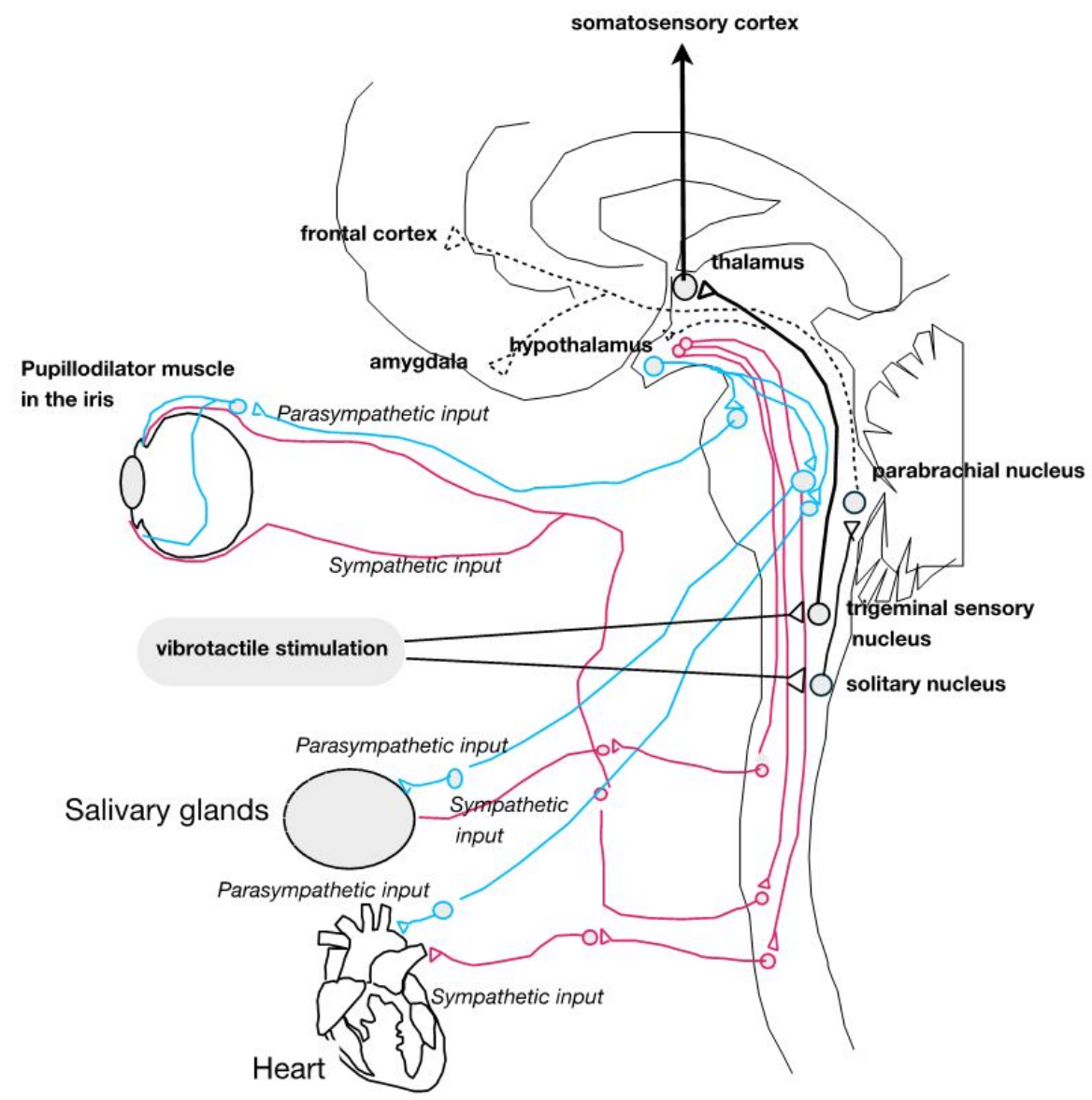

Figure 7. Pathways responsible for somatosensory information in the brain. Somatosensory information evoked by vibrotactile stimulation is relayed by the trigeminal sensory complex and solitary and parabrachial nuclei, which arrive at the hypothalamus, thalamus, amygdala, and frontal cortex, respectively. The autonomic system (particularly the parasympathetic nervous system) produces increased salivation. The lateral branch of the trigeminal sensory nucleus projects to the parabrachial nucleus. Information from the parabrachial nucleus is received by the amygdala and frontal cortex. Furthermore, somatosensory information is projected to the primary somatosensory cortex and relayed to the frontal cortex via the parietal association area. Thus, this information finally leads to a relaxed feeling, and BBF waves reflect parasympathetic activity (modified from schemas in Principles of Neural Science 2000b and Handbook of Neuropsychology 1994). 
The $89 \mathrm{~Hz}-\mathrm{S}$ stimulation evokes parasympathetic activity. This conclusion was based on increased salivation and heart rate and decreased pupil diameter during $89 \mathrm{~Hz}-\mathrm{S}$ vibrotactile stimulation. Increased parotid gland salivation may be due to appetite, and increased salivation in the submandibular and sublingual glands may be associated with feelings of calm. On the other hand, we examined changes in the HRV module (RR intervals) between the resting state and exposure to various stimuli, and we showed increased heart rates during $89 \mathrm{~Hz}-\mathrm{S}$ stimulation in comparison with the resting state (Billman, 2011). Furthermore, we think that the pupillary light-reflex test (Brashow, 1968) and heart rate HRV module (Billman, 2011) are the best tests for examining autonomic nervous system function (Gadner and Martin, 2000).

\section{Conclusion}

We showed that the most effective changes in salivation, pupil contraction, and HRV modulation (RR interval) were elicited by $89 \mathrm{~Hz}-\mathrm{S}$ vibrotactile stimulation on the face. We thus conclude that $89 \mathrm{~Hz}-\mathrm{S}$ vibrotactile stimulation affected parasympathetic activity based on changes observed in three organs. We also investigated autonomic activity by observing fNIRS waves. Because increased salivation was only observed in the submandibular and sublingual glands, it was likely not due to hunger. Furthermore, pupil constriction due to 89 $\mathrm{Hz}-\mathrm{S}$ stimulation was less than that due to light following the resting condition. This likely indicated parasympathetic activity induced by $89 \mathrm{~Hz}-\mathrm{S}$ stimulation. Changes in heart rate (RR intervals) during various stimuli were as effective as changes due to various stimuli combined with the $89 \mathrm{~Hz}-\mathrm{S}$ stimulation. BBF oxyHb concentrations in the frontal cortex during $89 \mathrm{~Hz}-\mathrm{S}$ vibrotactile stimulation were the same as those in subjects who preferred listening to classical music. Thus, $89 \mathrm{~Hz}-\mathrm{S}$ vibrotactile stimulation may produce relaxation; salivation increases, pupil diameter constricts, and the heart rate (RR interval) is prolonged due to parasympathetic excitation. Thus, we believe that fNIRS in the frontal cortex reflects autonomic activity.

\section{Acknowledgements}

This work was supported by a Sogoshigaku research grant and the Sato Fund, as well grants from the Ministry of Education and a Grant-in-Aid for Scientific Research (21592539).

\section{Author details}

Nelcy Hisao Hiraba ${ }^{1 *}$, Motoharu Inoue ${ }^{1}$, Takako Sato $^{2}$, Satoshi Nishimura ${ }^{2}$, Masaru Yamao$\mathrm{ka}^{3}$, Takaya Shimano ${ }^{1}$, Ryuichi Sampei ${ }^{1}$, Katuko Ebihara ${ }^{1}$, Hisako Ishii ${ }^{1}$ and Koichiro Ueda ${ }^{1}$

*Address all correspondence to: hiraba@dent.nihon-u.ac.jp 
1 Departments of Dysphasia Rehabilitation, Japan

2 Oral and Maxillofacial Surgery, Japan

3 Nihon University, School of Dentistry, 1-8-13 Kanda-surugadai, Chiyoda-ku, Japan

\section{References}

[1] Brashow, J. I. (1968). Pupillary changes and reaction time with varied stimulus uncertainty. Psychonomic Science, 13, 69-70.

[2] Burdette, B. H., \& Gale, E. N. (1988). The effects of treatment on masticatory muscle activity and mandibular posture in myofascial pain-dysfunction patients. J Dent Res, 67, 1126-1130.

[3] Chiang, C. Y., Hu, W., \& Sessle, B. J. (1994). Parabrachial area and nucleus raphe magnus-induced modulation of nociceptive and non-nociceptive trigeminal subnucleus caudalis neurons activated by cutaneous or deep inputs. J. Neurophysiol., 71, 2430-2445.

[4] Despopoulos, A., \& Silbernagl, S. (2003). Nutrition and digestion. In: Color Atlas of Physiology. 5 th ed. New York Medical Science International,Ltd. , 226-265.

[5] Gardner, E. P., \& Martin, J. H. (2000c). Coding of sensory information. In: Kandel ER., Schwartz JH., Jessell TM., editors, Principles of neuronal science, 4th ed, New York: McGraw-Hill , 411-429.

[6] Billman, George E. (2011). Heart rate variability-a historical perspective. Frontiers in Physiology, 1-13.

[7] Hiraba, H., Yamaoka, M., Fukano, M., Ueda, K., \& Fujiwara, T. (2008). Increased secretion of salivary glands produced by facial vibrotactile stimulation. Somatosensory and Motor Research, 25, 222-229.

[8] Hiraba, Hisao, Sato, Takako, Nishimura, Satoshi, Yamaoka, Masaru, Inoue, Motoharu, Sato, Mitsuyasu, Iida, Takatoshi, Wada, Satoko, Fujiwara, Tadao, \& Ueda, Koichiro. (2011). Changes in brain blood flow on frontal cortex depending on facial vibrotactile stimuli. In Vibration Analysis and Control-New Trends and Developments. Francisco Beltran-Carbajal ed. In Tech Croatia http://www.intechweb.org. , 337-352.

[9] Hoshi, Y., Kobayashi, N., \& Tamura, M. (2001). Interpretation of near-infrared spectroscopy signals: a study with a newly developed perfused rat brain model. J Appl Physiol., 90, 1657-1662.

[10] Ivarsen, S., Ivarsen, L., \& Saper, C. B. (2000b). The Autonomic nervous system and the hypothalamus. In: Kandel ER., Schwartz JH., Jessell TM., eds, Principles of Neuronal Science 4 th ed, New York McGraw-Hill. , 960-981. 
[11] Ivarsen, S., Kupfermann, F., \& Kandel, E. R. (2000a). Emotional states and feelings. In: Kandel ER., Schwartz JH., Jessell TM., editors, Principles of Neuronal Science, 4 th ed, New York McGraw-Hill , 982-997.

[12] Matuo, R. (2003). Daeki, Daekisen. In Nakamura Y., Morimoto T. And Yamada Y, editors, Basic Physiology for Dental Students, 4 th ed, Tokyo Ishiyaku Co. in Japanese), 381-398.

[13] Petrides, M. (1994). Frontal lobes and working memory: evidence from investigations of the effects of cortical excisions in nonhuman primates. In: Handbook of Neuropsychology, F. Boller and J. Grafman (eds.), Elsevier Science B.V., Amsterdam, , 9, 50-82.

[14] Sakatani, K., Lichty, W., \& Xie, Y. (1999). Effects of aging on language-activated cerebral blood oxygenation changes of the left prefrontal cortex. Near infrared spectroscopy study. J Stroke Cerebrovascular Dis., 8, 398-403.

[15] Sakatani, K., Yamashita, D., \& Yamanaka, T. (2006). Changes of cerebral blood oxygenation and optical path length during activation and deactivation in the prefrontal cortex measured by time-resolved near-infrared spectroscopy. Life Sciences, 78, 2734-2741.

[16] Scott, T. R., \& Small, D. M. (2009). The role of the parabrachial nucleus in taste processing and feeding. Ann NY Acad Sci., 1170, 372-377.

[17] Ueda, K. (2005). Sessyoku enge rehabilitation. In: Uematsu H, Inaba S, Watanabe M, editors. Koureishya Shika guidebook. Tokyo Ishiyaku in Japanese)., 248-275.

[18] Yamamoto, T., Takemura, M., Inui, T., Torii, K., Maeda, N., Ohmoto, M., Matumoto, I., \& Abe, K. (2009). Functional organization of the rodent parabrachial nucleus. Ann N Y Acad Sci., 1170, 378-382.

[19] Yamaoka, M., Hiraba, H., Ueda, K., \& Fujiwara, T. (2007). Development of a vibrotactile stimulation apparatus for orofacial rehabilitation. Nihondaigaku Shigakubu Kiyou, in Japanese), 35, 13-18. 
